\title{
Field Lessons from a Zoonotic Disease Study in the Nairobi Health Surveillance System
}

\author{
Djesika D. Amendah*, Peterrock Muriuki, Nicholas Ngomi, Martin kavao and Nelson Muhia \\ African Population and Health Research Center (APHRC), Nairobi, Kenya
}

\section{Objective}

Disseminate field lessons from a zoonotic disease study nested on the Nairobi Urban Health and Demographic Surveillance System (NUHDSS). The study investigates the emergence and introduction of zoonoses in urban areas

\section{Introduction}

About $60 \%$ of Nairobi residents live in slums with higher poverty, population density prevalence diseases and lower health access than the city average. Some residents own livestock or in are in contact with its products. Most slums dwellers work outside slums. Thus, health surveillance in slum area is vital because of potential disease outbreaks and spread. Yet, little is known on practice/challenges of health surveillance in resource-limited slums.

\section{Methods}

The African Population and Health Research Center established the NUHDSS in two slums (Korogocho and Viwandani) in 2003 and has since collected routine information on birth, deaths, cause of deaths, migration thrice a year. Currently, about 78000 persons living in 25,000 households are in NUHDSS. On this NUHDSS framework was nested a maternal and child health $(\mathrm{MCH})$ longitudinal study collecting socio-economic characteristics, childhood diseases, vaccination status, access to care, anthropometrics and other information among 3000 mother-child pairs. Babies $<7$ months born to resident mothers are recruited and followed-up till 5 years. The Urban Zoonoses case-control study was nested on the MCH. E.choli is used as examplar of emerging pathogen with multiple hosts. From August 2013 to April 2014, we collected additional information on hygiene practices, and diarrhea risk factors in the MCH study. Moreover, we collected fecal, food and water samples from about 200 children with diarrhea and 400 randomly selected controls to explore diarrhea-causing pathogens in laboratories.

\section{Results}

Here are the main lessons

Sustaining Community Participation: Most residents are poor and highly mobile and do not benefit individually from participation in the surveillance which could contribute to attrition. So we ensure their communities benefit by offering health camps with free care, upgrading health facilities, schools and by disseminating research results locally. We also use the NUHDSS platform for intervention research to improve knowledge while benefiting study participants.

Engaging Gatekeepers Some heads of local organizations, opinion leaders, etc. may act as gatekeepers blocking access to the community. We get their "buy-in" with sensitization, community leadership meetings to explain methods and objectives prior to, and during studies.

Hiring Local Fieldworkers We hire as fieldworkers educated slums residents who know local codes and people. We train and give education benefits for skills improvement and retention.

Engaging with Mothers The main respondents are women so we employ a female-only collection team. When respondents are casual workers with odd hours, interviews are conducted at a time and place convenient for mothers which stresses the importance of local fieldworkers and flexibility.

Keeping Employees and Material Safe Despite employing local residents, "no-go" areas exist. We hire additional security to accompany fieldworkers to collect data there and when bulky equipment is used for anthropometrics.

Collecting Sample Obtaining samples was easier from children with diarrhea than from the control group. We dedicated fieldworkers to collect sample among the latter and carry them to the labs.

Offering Clinical Care Clinical officers using lab results offer treatment to children with diarrhea, and others in all households involved in the zoonotic study and referral when needed. These services increase the community's acceptance of the study

\section{Conclusions}

Accounting for local context is vital for a successful population surveillance system and the zoonotic study nested on it.

\section{Keywords}

Zoonotic disease; Health Surveillance; Low-ressource; Kenya

\section{Acknowledgments}

Medical Research Council, Natural Environment Research Council, Economic and Social Research Council, Biotechnology and Biosciences Research Council for funds received through the Environmental \& Social Ecology of Human Infectious Diseases Initiative (ESEI), Grant G1100783/1.

William and flora Hewlett foundation, Bill and Melinda Gates foundation, and Sweden International Development Cooperation Agency

\section{References}

Emina J, Beguy D, Zulu EM, Ezeh AC, Muindi K, et al. (2011) Monitoring of health and demographic outcomes in poor urban settlements: evidence from the Nairobi Urban Health and Demographic Surveillance System. J Urban Health 88 Suppl 2: S200-218.

APHRC (2002) Population and Health Dynamics in Nairobi's Informal Settlements. Nairobi, Kenya: African Population and Health Research Center.

\author{
*Djesika D. Amendah \\ E-mail: damendah@aphrc.org
}

